\title{
Improved damage quantification method based on substructure modal strain energy
}

\author{
H. Fang \\ China Academy of Engineering Physics, \\ Institute of Structural Mechanics, China
}

\begin{abstract}
This paper presents an improved method for the damage severity estimate for lattice structures based on the employment of the substructure modal strain energy (SMSE). The significant advantage associated with the improved method over traditional modal energy methods is that it can directly use the mode shape of the damaged element to quantify damage severity exactly. Additionally, the new method does not require the analytical and measured modes to be consistent in scale, or to be normalized. Numerical studies in this paper are conducted for a three-dimensional frame structure based on synthetic data generated from finite element models.
\end{abstract}

Keywords: damage quantification, substructure energy, modal analysis.

\section{Introduction}

The properties of the truss material that appear most attractive are those that govern the use of the truss as cores for sandwich structures as having lower weight than competing materials, and potentially superior heat dissipation, vibration control and energy dissipation characteristics [1]. The mechanical properties of lattice structures may degrade severely in the presence of damage. Structural damage often occurs in one or more elements of a structure with a loss of stiffness. These changes in structural properties in turn alter the dynamic response behaviour of the structure from its pre-damage condition. Therefore, it is common to monitor structural health condition using data obtained from an associated vibration testing.

A number of approaches to the damage severity quantification exist [2]. The modal testing and analysis method seeks to determine the modal parameters, 
including natural frequencies and mode shapes, from the measured responses, and then detect damaged element from these data, or their combination, such as modal strain energy (MSE). Traditionally, the damage detection methods based on modal data can be classified into two major groups: iterative methods and non-iterative methods [3]. Almost all of damage quantification methods base on modal strain energy $[4,5]$ belong to the first of these two groups. For iterative methods, spatially complete measured modes is not necessary, but there are some disadvantages: possibility of solutions being divergent, demand on excessive computational time, requirement to pair measured and analytical modes, requirement to scale measured and analytical modes [6]. To avoid these problems, $\mathrm{Hu}$ et al. [7] develop a non-iterative MSE-based method which can exactly quantify the severity of the damage in the 3D frame structures. However, the existing non-iterative method requires the information of spatial complete modes measured from the damaged structure. In practice, the degrees of freedom of the structure are usually very large, and also only a limited number of the lowest vibration modes of the system can be measured accurately. Many techniques that appear to work well in example cases actually perform poorly when subjected to the measurement constraints imposed by actual testing. Techniques that are to be seriously considered for implementation in the field should demonstrate that they can perform well under the limitations of a small number of measurement locations [2].

An improved damage severity quantification method is developed in this paper based on the substructure modal strain energy. The improved algorithm can exactly quantify damage severity only using the mode shape of the damaged member in the structures.

\section{Substructure modal strain energy method}

The structure of the lattice beam is composed of a sequence of identical unit cells. Each cell is composed of beam elements assembled to form a lattice structure. The equivalent system with repetition characteristic is shown in Fig. 1.

(A)

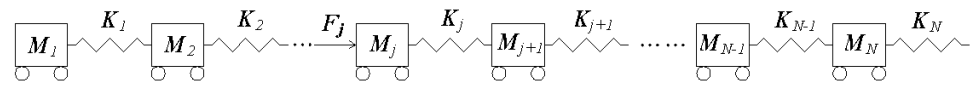

(B)

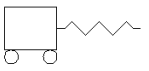

Figure 1: $\quad$ Equivalent system (A) unit (B).

The total modal strain energy of the structure considered may be expressed as

$$
\mathrm{E}=\sum_{j=1}^{N} \varepsilon_{j},
$$


in which $\varepsilon_{j}$ denotes the modal strain energy of the $j$ th substructure and $N$ denotes the total number of substructures. The substructure modal strain energy (SMSE) may be defined as

$$
\varepsilon_{j}=\boldsymbol{\Phi}_{j}^{T} \mathbf{K}_{j} \boldsymbol{\Phi}_{j},
$$

where $\mathbf{K}_{j}$ and $\boldsymbol{\Phi}_{j}$ denote the stiffness matrix of the $j$ th substructure and the eigenvector for the $j$ th substructure respectively, and superscript $T$ is used to indicate transpose operator. The stiffness matrix of the substructure is

$$
\mathbf{K}_{j}=\mathbf{K}_{s u b} \text {. }
$$

For repetitive structure, the eigenvector $\boldsymbol{\Phi}_{j}$ is made up of the left and right nodal displacement vectors

$$
\boldsymbol{\Phi}_{j} \equiv\left\{\boldsymbol{\Phi}_{L} \quad \boldsymbol{\Phi}_{R}\right\}_{j}^{T}
$$

in which $\left\{\boldsymbol{\Phi}_{L}\right\}_{j}$ and $\left\{\boldsymbol{\Phi}_{R}\right\}_{j}$ denote the nodal displacement vectors on the common boundary between the $j-1, j+1$ and $j$ th substructures, respectively. The continuity condition may be expressed

$$
\left\{\boldsymbol{\Phi}_{L}\right\}_{j+1}=\left\{\boldsymbol{\Phi}_{R}\right\}_{j}, j=1,2, \cdots, N
$$

Assume the stiffness matrix $\mathbf{K}_{j}^{d}$ of the substructure of the damaged structure is modification of $\mathbf{K}_{j}$.

$$
\mathbf{K}_{j}^{d}=\mathbf{K}_{j}-\sum_{z=1}^{D_{j}} \alpha_{j, z} \mathbf{K}_{j, z}, \quad 0<\alpha_{j, z} \leq 1,
$$

in which $\mathbf{K}_{j, z}$ is the stiffness of the $z$ th element in the $j$ th substructure, and $\alpha_{j, z}$ is the correction factors of the stiffness matrix, $D_{j}$ is the total number of damaged elements in the $j$ th substructure. Superscript $d$ is used to indicate damage version.

A residual force is used in the present formulation to simulate modification of stiffness matrix

$$
\mathbf{F}_{j}^{R}=\sum_{z=1}^{D_{j}}\left(\alpha_{j, z} \mathbf{K}_{j, z}\right) \boldsymbol{\Phi}_{j}^{d}
$$

where $\boldsymbol{\Phi}_{j}^{d}$ denote the eigenvector for the $j$ th damaged substructure. We get the substructure modal strain energy of damaged substructure

$$
\varepsilon_{j}^{d}=\boldsymbol{\Phi}_{j}^{d T} \mathbf{K}_{s u b} \boldsymbol{\Phi}_{j}^{d}-\frac{1}{2} \boldsymbol{\Phi}_{j}^{d T} \mathbf{F}_{j}^{R} .
$$

Applying the U-transformation [8] to $\boldsymbol{\Phi}_{j}^{d}$ and $\mathbf{F}_{j}^{R}$ 


$$
\boldsymbol{\Phi}_{j}^{d}=\frac{1}{\sqrt{N}} \sum_{k=1}^{N} e^{i(j-1) k \psi} \mathbf{q}_{k}, \quad \mathbf{F}_{j}^{R}=\frac{1}{\sqrt{N}} \sum_{k=1}^{N} e^{i(j-1) k \psi} \mathbf{f}_{k},
$$

in which $\psi=2 \pi / N$ and $i=\sqrt{-1}$, substituting into Eqs. (7) (8) (9) and (1), we get the substructure modal strain energy $\mu_{k}^{d}$ of the damaged structure in Udomain

$$
\mu_{k}^{d}=\mathbf{q}_{k}^{T} \mathbf{K}_{s u b} \mathbf{q}_{k}-\frac{1}{2} \mathbf{q}_{k}^{T} \mathbf{f}_{k}^{R},
$$

the continuity condition

$$
\mathbf{q}_{k, R}=e^{i k \psi} \mathbf{q}_{k, L}
$$

The inertia force $\omega^{d 2} \mathbf{M}_{s u b} \boldsymbol{\Phi}_{j}^{d}$ ( $\omega^{d}$ is the corresponding eigenvalue) is added into system as loading vector. Damping is not considered in this paper. By the equation of variation, one gets

$$
\left(\mathbf{K}_{s u b}^{*}-\omega^{d 2} \mathbf{M}_{s u b}^{*}\right) \mathbf{q}_{k, L}=\mathbf{f}_{k}^{*},
$$

in which $\mathbf{K}_{\text {sub }}^{*}=\overline{\mathbf{T}}_{k}^{T} \mathbf{K}_{\text {sub }} \mathbf{T}_{k}, \quad \mathbf{M}_{s u b}^{*}=\overline{\mathbf{T}}_{k}^{T} \mathbf{M}_{s u b} \mathbf{T}_{k}, \quad \mathbf{f}_{k}^{*}=\overline{\mathbf{T}}_{k}^{T} \mathbf{f}_{k}$, $\mathbf{T}_{k}=\left[\begin{array}{ll}\mathbf{I}_{L} & e^{i k \psi} \mathbf{I}_{R}\end{array}\right]^{T}$, and the superior bar means the equation with complex variables. Applying the inverse U-transformation

$$
\mathbf{q}_{k}=\frac{1}{\sqrt{N}} \sum_{j=1}^{N} e^{-i(j-1) k \psi} \mathbf{\Phi}_{j}^{d}, \mathbf{f}_{k}=\frac{1}{\sqrt{N}} \sum_{j=1}^{N} e^{-i(j-1) k \psi} \mathbf{F}_{j}^{R},
$$

we get the expanded governing equations for free-vibration of the damaged structure

$$
\boldsymbol{\Phi}_{j}^{d}=\sum_{p=1}^{N_{d}} \mathbf{H}_{j, p} \sum_{z=1}^{D_{p}} \alpha_{p, z} \mathbf{K}_{p, z} \boldsymbol{\Phi}_{p}^{d}+\sum_{b=1}^{N_{b}} \mathbf{H}_{j, b} \mathbf{K}_{b} \boldsymbol{\Phi}_{b}^{d}
$$

in which $\mathbf{H}_{j, x}=\frac{1}{N} \sum_{k=1}^{N} e^{i(j-x) k \psi} \mathbf{T}_{k}\left(\mathbf{K}_{s u b}^{*}-\omega^{d 2} \mathbf{M}_{s u b}^{*}\right)^{-1} \overline{\mathbf{T}}_{k}^{T}, j=(1, \ldots, N), N$ is the number of substructures, $N_{d}$ is the number of damaged substructures. In general, a structure has some other disordered substructures, such as the border substructures or poling units, and then these disordered terms should be added as the loading for the structure. $N_{b}$ is the number of border substructures. According to Eq. (12), we get

$$
\boldsymbol{\Phi}_{j}^{d}=\sum_{p=1}^{N_{d}} \sum_{z=1}^{D_{p}} \alpha_{p, z} \mathbf{B}_{j, p z} \boldsymbol{\Phi}_{p, z}^{d}
$$

in which 


$$
\mathbf{B}_{j, p z}=\mathbf{H}_{j, p} \mathbf{K}_{p, z} \boldsymbol{\Phi}_{p}^{d}+\sum_{b=1}^{N_{b}} \mathbf{H}_{j, b} \mathbf{K}_{b}\left(\mathbf{I}_{b}-\sum_{b=1}^{N_{b}} \mathbf{H}_{b, b} \mathbf{K}_{b}\right)^{-1} \mathbf{H}_{b, p} \mathbf{K}_{p, z},
$$

and $\boldsymbol{\Phi}_{p, z}^{d}$ is the eigenvector of the damaged member. Pre-multiplying the equation in the Eq. (13) by $\boldsymbol{\Phi}_{p, z}^{T}$ (eigenvector of the corresponding member in the undamaged structure), and using a new index $Z$ to replace $(p, z)$, we get

$$
\gamma_{Z}=\sum_{Z=1}^{N_{D}} c_{Z} \alpha_{Z}
$$

where

$$
\gamma_{Z}=\boldsymbol{\Phi}_{p, z}^{T} \boldsymbol{\Phi}_{p, z}^{d}, c_{Z}=\boldsymbol{\Phi}_{p, z}^{T} \mathbf{B}_{p, p z} \boldsymbol{\Phi}_{p, z}^{d}, N_{D}=\sum_{p=1}^{N_{d}} D_{p} .
$$

Written in a matrix form, one has $\boldsymbol{\Gamma}=\mathbf{C} \boldsymbol{\alpha}$ in which $\mathbf{C}$ is $N_{E} \times N_{D}$ matrix, $\boldsymbol{\Gamma}$ is column vector of size $N_{E}$, and $\boldsymbol{\alpha}$ is column vectors of size $N_{D}$. It is worthy to mention that those modes $\boldsymbol{\Phi}$ and $\boldsymbol{\Phi}^{d}$ of the undamaged and damaged structures can be arbitrary modes of the baseline and true structures in the sense that they are not required to start from the first mode and be the matching modes.

An estimate for correction factors, denoted $\widehat{\boldsymbol{\alpha}}$, is obtained as

$$
\widehat{\boldsymbol{\alpha}}=\left(\mathbf{C}^{\mathrm{T}} \mathbf{C}\right)^{-1} \mathbf{C}^{T} \boldsymbol{\Gamma} .
$$

The core of the improved algorithm is to formulate simultaneous linear equations associated with substructure modal strain energy (SMSE). The significant advantage of the SMSE method over other non-iterative methods is that the spatially complete mode shape is not needed. Additionally the improved algorithm does not require the analytical and measured modes to be consistent in scale, or to be normalized.

\section{Numerical examples}

Numerical examples are given below to demonstrate the accuracy of the SMSE method for 3D lattice structures. The considered structure features a beam-type lattice with elements arranged according to a square configuration as shown in Fig. 2, where each structural member is modeled as a three-dimensional uniform beam element and is distinguished by assigning an element number. This structure has the same configuration as structure used by Hu et al. [7]. The length of all horizontal members oriented in the $\mathrm{x}$ direction is $1 \mathrm{~m}$, all horizontal members oriented in the y direction $3 \mathrm{~m}$, and all vertical members $1 \mathrm{~m}$. Young's modulus $\mathrm{E}$ is a constant equal to $2.1 \times 10^{11} \mathrm{~Pa}$ for all members before damage, the cross-section area and the associated moment of inertia for all members are $\mathrm{A}=2.825 \times 10^{-3} \mathrm{~m}^{2}$ and $\mathrm{I}=2.89 \times 10^{-6} \mathrm{~m}^{4}$, respectively. All computations in this paper were done in Matlab. 
Three particular tasks are to be performed. Task 1 considers three singledamage scenarios, with 5\% stiffness loss at a column (element 18), a long-span beam (element 22), and a short-span beam (element 23), respectively. Task two considers the same single damage scenarios investigated in Task one, but under the assumption that the measured mode shapes are contaminated by uncorrelated random errors. Assuming that two damaged elements presented in Task one take place simultaneously in the structure, Task three investigates the capability of the method on quantifying severity for multiple damaged elements. Performing the eigen-analysis, one obtains the mode frequencies and shapes of the undamaged and damaged structures. Table 1 summarizes the five single and multiple damage scenarios. Listed also are the first five frequencies of the undamaged and the five damaged structures. Fig. 3 shows the first five mode shapes of the undamaged structure.

Table 1: $\quad$ A summary of the undamaged and damaged structures.

\begin{tabular}{cccccccc}
\hline \multirow{2}{*}{ Structure } & \multirow{2}{*}{$\begin{array}{c}\text { Damaged } \\
\text { element }\end{array}$} & \multirow{2}{*}{$\begin{array}{c}\text { Stiffness } \\
\text { Loss }\end{array}$} & \multicolumn{5}{c}{ Frequency } \\
\cline { 4 - 8 } & & & 1st & 2nd & 3rd & 4th & 5th \\
\hline undamaged & None & $0 \%$ & 6.9105 & 9.3615 & 11.2781 & 23.0373 & 29.3154 \\
A & 18 & $5 \%$ & 6.9084 & 9.3561 & 11.2719 & 23.0252 & 29.3037 \\
B & 22 & $5 \%$ & 6.8958 & 9.3615 & 11.2765 & 23.0074 & 29.3151 \\
C & 23 & $5 \%$ & 6.9105 & 9.3480 & 11.2632 & 23.0373 & 29.2786 \\
D & $18 \& 22$ & $5 \%$ & 6.8938 & 9.3561 & 11.2703 & 22.9950 & 29.3034 \\
E & $22 \& 23$ & $5 \%$ & 6.8958 & 9.3480 & 11.2616 & 23.0074 & 29.2783 \\
\hline
\end{tabular}

(A)

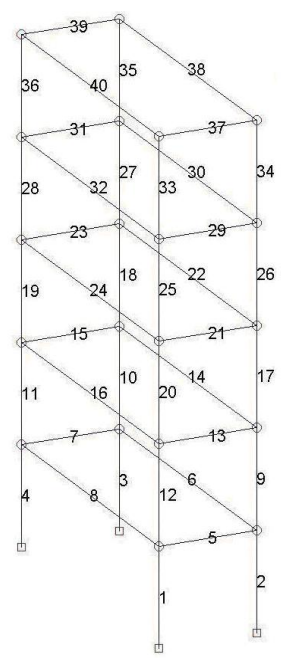

(B)

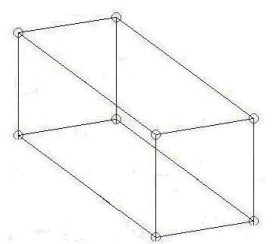

Figure 2: $\quad$ The sketch of the structure (A) and substructure (B). 

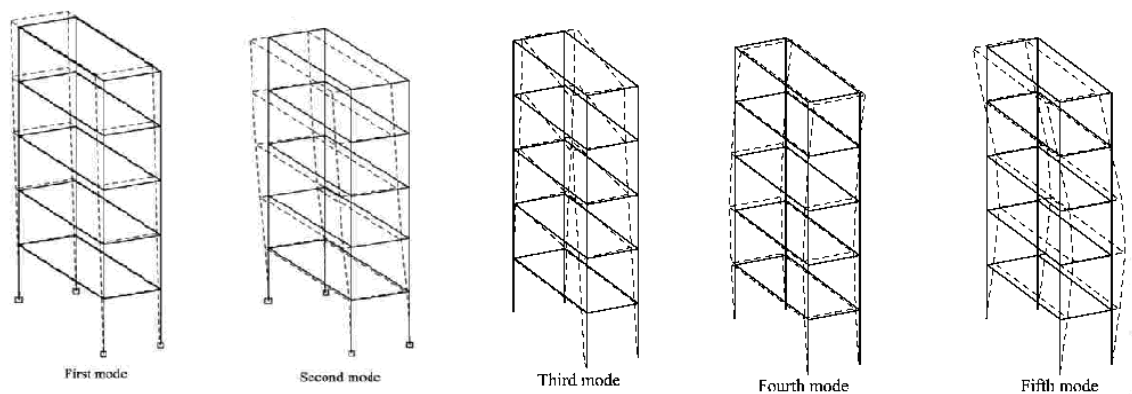

Figure 3: $\quad$ The first five modes of the undamaged structure.

\subsection{Single damage scenarios without noise}

In task one, three single damage scenarios (structure $\mathrm{A} \sim \mathrm{C}$ ) shown in Table 1 are performed. According to Eq. (13), the measured modes only need the DOFs of the damaged elements. Taking the first mode for both the undamaged and damaged structures, i.e., $m^{b}=m^{d}=(1)$ (hereafter $m^{b}$ and $m^{d}$ indicate the mode number for the undamaged and the damaged structures, respectively); one exactly obtains the damage severity $\widehat{\boldsymbol{\alpha}}=5 \%$. One also gets accurate results $\hat{\boldsymbol{\alpha}}$ for damage at element 22, and 23 using the SMSE method. When the damaged elements occur in any substructure, one also yields the correct results $\widehat{\boldsymbol{\alpha}}$ using any mode for both the undamaged and damaged structures.

\subsection{Single-damage scenarios with noise}

Due to unavoidable errors in instrumentation and measurements, measured data are often contaminated with noise. The random error (modal noise) of the measured mode shape is quantified in percentage. Herein a modal noise level of $5 \%$ indicates the standard deviation of the random error is $5 \%$ of the true modal value at each corresponding coordinate. Task two considers three single damage scenarios (structure $\mathrm{A} \sim \mathrm{C}$ ). A 5\% change in several entries of each eigenvector was randomly introduced [7]. This error propagation from the measured mode shapes to the estimate of the severity will be studied by using Monte Carlo simulations.

The goodness of the estimate for the damage severity is judged based on both statistical accuracy and precision. The biased percentage error (a statistical measure of accuracy) of the estimated damage severity [7], denoted by

$$
\varsigma_{b}=\left|\frac{\bar{\alpha}-\alpha}{\alpha}\right| \times 100 \%,
$$

where $\bar{\alpha}$ is the sample mean of the estimated damage extent from simulations, $\alpha$ is the true damage severity. The random percentage error (a statistical measure of precision) of the estimated damage severity [7], denoted by 


$$
\varsigma_{r}=\left|\frac{\sigma_{\alpha}}{\bar{\alpha}}\right| \times 100 \%,
$$

in which $\sigma_{\alpha}$ is sample standard deviation of the estimated $\alpha$ computed from simulations.

5000 Monte Carlo simulations are always repeated for each case under investigation. The measured modes only need the DOFs of the damaged elements. One takes from the 1 st to the 5 th mode respectively for both the undamaged and damaged structures, i.e., $m^{b}=m^{d}=(1,2, \ldots$, or 5). Table 2 provides the simulated results of $\zeta_{b}$ and $\zeta_{r}$ for the five exercises, using the SMSE method. For the damaged structures B and C, taking any mode, the results of $\zeta_{b}$ and $\varsigma_{r}$ is statistically good. For the damaged structure A, taking the first three modes, it is noticed that the modal noise has a much larger effect on the accuracy and precision of the severity quantification for a column damage than on that for beam damages. While one takes the 4th, or 5 th mode, the results of $\zeta_{b}$ and $\zeta_{r}$ is statistically good. Notice that the 4 th and 5 th modes have distinct deformation in the substructures. Then this can attribute to the fact that damage typically is a local phenomenon, and local response is captured by higher order modes whereas lower order modes tend to capture the global response of the structure and are less sensitive to local changes in a structure. Testing on structures having damage in different substructures also yields the similar results.

From the above numerical exercise, a few guidelines can be summarized. First, it is good to use any modes for any damaged beam. Second, it is required to choose the modes that have noticeable local response for damaged columns.

Table 2: $\quad$ Results of biased and random percentage errors of single-damage severity estimate on 5\% modal noise level, using SMSE.

\begin{tabular}{ccrrccccc}
\hline & & \multicolumn{2}{c}{ Structure A } & \multicolumn{2}{c}{ Structure B } & \multicolumn{2}{c}{ Structure C } \\
\cline { 3 - 9 } Exercise & Modes & \multicolumn{2}{c}{ Element 18 } & \multicolumn{2}{c}{ Element 22 } & \multicolumn{2}{c}{ Element23 } \\
& & \multicolumn{1}{c}{$\boldsymbol{S}_{b}$} & \multicolumn{1}{c}{$\boldsymbol{\zeta}_{r}$} & $\boldsymbol{S}_{b}$ & $\boldsymbol{S}_{r}$ & $\boldsymbol{S}_{b}$ & $\boldsymbol{S}_{r}$ \\
\hline & & $m^{b}=m^{d}=1$ & 80.13 & 5624.70 & 0.03 & 4.95 & 0.12 & 4.53 \\
1 & $m^{b}=m^{d}=2$ & 994.73 & 8204.50 & 0.10 & 4.75 & 0.17 & 4.66 \\
3 & $m^{b}=m^{d}=3$ & 20.77 & 499.22 & 0.23 & 5.65 & 0.13 & 4.57 \\
4 & $m^{b}=m^{d}=4$ & 0.58 & 7.10 & 0.03 & 5.76 & 0.13 & 39.32 \\
5 & $m^{b}=m^{d}=5$ & 1.54 & 12.99 & 0.12 & 7.09 & 0.14 & 10.89 \\
\hline
\end{tabular}

\subsection{Multiple-damage scenarios}

Two multi-damage scenarios (structure $\mathrm{D} \sim \mathrm{E}$ ) as shown in Table 1, are investigated. Again, all damaged members are with 5\% loss of stiffness. For structures of two damaged elements, one takes the first mode for both the undamaged and damaged structures, i.e., $m^{b}=m^{d}=(1)$, and the measurement locations include the damaged elements, then, one obtains the exact damage severity for all damage elements under the noise-free measurement condition. 
Table 3: $\quad$ Results of biased and random percentage errors of multiple-damage severity estimate on 5\% modal noise level, using SMSE.

\begin{tabular}{|c|c|c|c|c|c|c|c|c|c|}
\hline \multirow{3}{*}{ Exercise } & \multirow{3}{*}{ Modes } & \multicolumn{4}{|c|}{ Structure D } & \multicolumn{4}{|c|}{ Structure E } \\
\hline & & \multicolumn{2}{|c|}{ Element 18} & \multicolumn{2}{|c|}{ Element 22} & \multicolumn{2}{|c|}{ Element 22} & \multicolumn{2}{|c|}{ Element 23} \\
\hline & & $\varsigma_{b}$ & $\zeta_{r}$ & $\zeta_{b}$ & $\zeta_{r}$ & $\zeta_{b}$ & $\zeta_{r}$ & $\varsigma_{b}$ & $\zeta_{r}$ \\
\hline 1 & $m^{b}=m^{d}=1$ & $6.61 \mathrm{e} 7$ & $2.25 \mathrm{e} 3$ & $2.95 \mathrm{e} 3$ & $6.31 \mathrm{e} 3$ & $1.04 \mathrm{e} 5$ & $6.98 \mathrm{e} 3$ & 2.52 & 189.32 \\
\hline 2 & $m^{b}=m^{d}=2$ & 173.43 & $1.39 \mathrm{e} 3$ & $2.13 \mathrm{e} 3$ & $1.92 \mathrm{e} 3$ & $1.56 \mathrm{e} 3$ & $5.63 \mathrm{e} 3$ & $1.68 \mathrm{e} 6$ & $5.29 \mathrm{e} 3$ \\
\hline 3 & $m^{b}=m^{d}=3$ & 191.99 & $1.03 \mathrm{e} 4$ & 360.57 & $2.75 \mathrm{e} 4$ & 317.62 & $1.06 \mathrm{e} 4$ & $2.89 \mathrm{e} 3$ & $6.96 \mathrm{e} 3$ \\
\hline 4 & $m^{b}=m^{d}=4$ & $1.62 \mathrm{e} 3$ & $2.39 \mathrm{e} 4$ & 12.57 & 440.36 & $5.05 \mathrm{e} 5$ & $1.47 \mathrm{e} 4$ & 311.00 & $9.97 \mathrm{e} 3$ \\
\hline 5 & $m^{b}=m^{d}=5$ & 252.19 & 747.89 & 554.25 & $1.46 \mathrm{e} 3$ & 6.38 & 168.60 & $1.06 \mathrm{e} 3$ & $2.14 \mathrm{e} 3$ \\
\hline 6 & $m^{b}=m^{d}=(1 \sim 3)$ & 10.88 & 98.00 & 26.66 & 366.90 & 5.72 & 22.29 & 2.19 & 54.86 \\
\hline 7 & $m^{b}=m^{d}=(1 \sim 5)$ & 6.62 & 10.60 & 7.47 & 15.62 & 0.11 & 4.47 & 0.20 & 5.28 \\
\hline
\end{tabular}

The same multiple-damage scenarios investigated above with the contaminated mode shapes are considered. Modal noise level is 5\%, and all simulated damages are with 5\% stiffness loss. Throughout this study, 5000 Monte Carlo simulations are always repeated for each case under investigation. One takes from the 1 st to the 5 th mode respectively, i.e., $m^{b}=m^{d}=(1,2, \ldots$, or 5). Table 3 provides the simulated results of $\varsigma_{b}$ and $\varsigma_{r}$ for the exercises. Then, the modes $1 \sim 3$ or $1 \sim 5$, i.e., $m^{b}=m^{d}=(1 \sim 3)$ or $(1 \sim 5)$, were taken. Table 3 provides the simulated results of $\zeta_{b}$ and $\zeta_{r}$ for the exercises too. Using multiple-modes for structures $(\mathrm{D} \sim \mathrm{E})$, the results of damage severity estimation are statistically better than single mode. From the results of these exercises, one observes that additional modes improve the reliability of the solution. Testing on structures having a damaged element at other substructures also yields the same conclusion. Due to the complicated mutual influence among multiple damaged members, modes associated with the modal noise effect on each damaged member are not easy to extract. The newly proposed method can accurately quantify the multiple damaged elements when the mode shapes are not contaminated. This method also can successfully quantify the damage severity based on the preset damage locations when the mode shapes are contaminated.

\section{Concluding remarks}

A newly developed non-iterative damage identification method, substructure modal strain energy (SMSE), which is capable of estimating for lattice core structures. In addition to its accuracy, the significant advantage over other modal energy methods is that the complete mode shape can be used directly. The method has the potential to be employed for plane structures as well. However, further development is still needed. Developing the SMSE method for plane structures is currently underway.

\section{References}

[1] A.G. Evans, J.W. Hutchinson, N.A. Fleck, M.F. Ashby, H.N.G. Wadley, The topological design of multifunctional cellular metals, Progress in Materials Science 46 (2001) 309-327. 
[2] S. W. Doebling, C. R. Farrar, M. B. Prime, D. W. Shevitz, Damage identification and health monitoring of structural and mechanical systems from changes in their vibration characteristics: A literature review, Los Alamos National Laboratory Report LA-13070-MS, Los Alamos, New Mexico, 1996.

[3] M. I. Friswell, J. E. Mottershead, Finite element Model Updating in Structural Dynamics, Kluwer Academic Publishers, Dordrecht, 1995.

[4] Z.Y. Shi, S.S. Law, L.M. Zhang, Improved Damage Quantification from elemental Modal Strain Energy Change, Journal of Engineering Mechanics 128 (5) (2002) 521-529.

[5] J.T. Kim, N. Stubbs, Improved damage identification method based on modal information, Journal of Sound and Vibration 252 (2) (2002) 223-238.

[6] S.J. Hu, H. Li, Simultaneous Mass, Damping, and Stiffness Updating for Dynamic Systems, AIAA Journal 45 (10) (2007) 2529-2537.

[7] S.J. Hu, S. Wang, H. Li, Cross modal strain energy method for estimating damage severity, ASCE Journal of Engineering Mechanics 132(4) (2006) 429-437.

[8] H.C. Chan, C.W. Cai, Y.K. Cheung, Exact Analysis of Structures with Periodicity using U-transformation, World Scientific, Singapore, 1998. 